\title{
Sketch Based Data Retrieval Using Reversible Data Hiding on Images
}

\author{
D.O.I - 10.51201/12511 \\ https://doi.org/10.51201/12511 \\ Dipika Birari $^{1}$, Dilendra Hiran ${ }^{2}$, Vaibhav Narawade ${ }^{3}$ \\ ${ }^{1}$ Research Scholar, Faculty of Computer Engineering, Pacific Academy of Higher Education and Research \\ University, Udaipur, India. \\ ${ }^{2}$ Pacific Institute of Computer Application, Udaipur, India. \\ ${ }^{3}$ Department of Computer Engineering, Ramrao Adik Institute of Technology, Navi Mumbai, India.
}

\begin{abstract}
Sketch Based Image Retrieval as name suggests is a branch of image retrieval that searches the images based on sketches. The sketches bridge the gap between imagination and real images. Sketches act as powerful searching tool in image retrieval. It efficiently uses the data hiding technique, which is a watermarking technique and can be used for embedding messages into images. A message can be embedded into color images without changing its grayscale version. Embedding of messages was thus done using unchanged gray version. It can also extract the messages. This unchanged grayscale version is maintained from message embedding to extraction process. This concept of watermarking generates SBIDR valuable, because many applications or algorithms for color images are based on its corresponding grayscale version. Embedding of data is carried out into in three-color channels of color image and in gray value of Sketch Image. Sketch image is encrypted with key, which will be required at the time of message extraction process after SBIR. In this way grayscale invariance is maintained which made the Sketch Based Retrieval more effective.
\end{abstract}

Keywords: Sketch based, Grayscale, Color channel, Invariance, Embedding Process, Extraction Process.

\section{INTRODUCTION}

Imaginations are often represented through imagery. Image is a thoughtful process on which we perform transformations, operations or apply various processing techniques. But during these transformations or processing, it should maintain its properties as well. Gray scale or Gray version is one of the important and powerful aspects of image processing, because gray images can be processed faster than the color images due to its low intensities. Whenever sketches are used to search images, the grayscale invariance is incredibly important because sketches are represented with Black and White color and other comparable images are colorful. Data is embedded into color images during which Red, Green and Blue colors channels are embedded with messages and sketches are embedded with the key. With this SBIR data hiding technique are often applied on images, which is to be studied.

It can be a useful application in police, military and crime system where sketch is employed as major factor to search for the criminals. And in such applications, reduction in processing accuracy may involve critical loss. Therefore, image retrieving and message retrieving, both are equally important. Further the CBIR, SBIR act as a powerful search tool that can be used as a supplementary tool for text based search. In SBIR, images are searched by sketches by using preprocessing, edge extraction, descriptor generation and constraints application to pick out strong or shaping edges. HoG\& GF-HoG descriptors are specially used for SBIR apart from this the HLR (Histogram Line Relationship) Descriptor [1] is employed to bridge the gap between line segments that offers the link between line segments. However to bridge the big appearance gap between sketches \& real colorful images; only edge extraction isn't satisfactory. Therefore it goes through first pre-processing where canny edge detection is employed for removal of noisy edges. Then with the assistance of descriptor \& edge extraction process, the pictures are extracted by applying some constraints. 
With this method, RDH [2] are often applied on real image during which one could extract the data hidden in the image. It is a fragile technique which is employed for authentication. Many RDH algorithms are categorized like histogram shift or lossless compression technique or expansion difference technique. This algorithm works on gray-scale of given image. However, the color images have more powerful visualization than gray images and multiple algorithms are present for performing the watermarking on color images like blue-channel based, 3-color channel based [26]. But these algorithms destroy their color images irreversibly i.e. the generated mark image appears to be a noisy image and differs from the original image. The sting extraction or feature extraction process gets further tormented by marked image. There are descriptors like SIFT, HoG which works on gray images as feature descriptor and works only on the luminance channel by converting color images into gray images. There are some RDH schemes in the color image that may be employed in SBIR color image to avoid interference with further image processing with the help of gray-scale invariance. So in this study, the RDH is used for processing with gray-scale invariance and to retrieve data from images so that marked images are used for further image retrieval through sketches.

\section{RELATED WORK}

Loss less Data embedding technique [3] accustomed to remove distortion, which occurs because of embedding in marked images. It works on image format which is most typical like JPEG (transform format), BMP (un-compressed format), PNG and GIF (pallet formats). It demonstrates the fragile technique to attain different tasks and authentication. Difference of expansion in data embedding [4] aimed to achieve high embedding capacity that contains redundancy technique in images to create low distortion rate. By using reversibility, original content was restored completely. Luminance Channel based Algorithms uses vision based masking [5] and quantization [6].Vision based masking model evaluates the robustness by density of messages which depends upon sensitivity and masking behaviour of the human sensory system. Whereas in quantization process, bit embedding is completed by single luminance value.

In Chrominance-channel based algorithm [7], watermark is embedded in chrominance channel to attain prime quality of watermarked videos and by using same key it can be extracted. 3-color channel based algorithm [8, 9] used Coefficient quantization and partitioning of images respectively. Coefficient quantization used for watermarking on color images which hide marking within 3 color channels with none distortion. In second approach it divides color image into patches (non-overlapped) and further their decomposition is calculated. In blue channel based algorithm [10] modulation by changing blue channels pixel value was performed. Gaussian mask is employed for equalization of intensity (luminance) and retrieving process is predicated in prediction system by using two more bits for a reference. Reversible Data Hiding for color images works on genetic algorithm for bitmap [11], Prediction error system [12] and payload partitioning [13]. First approach is employed to search for bitmap. These bitmaps are used for block compressing. And to attain reversibility, quantization level is employed. In the second approach, the independent message embedding is finished in every channel. It is a supported prediction-error system to extend efficiency of algorithm. This RDH works on the color images. Whereas in third approach, payload portioning has importance which is embedded into every channel. This RDH works on inter- channel correlation by payload partitioning and embedding process per each of its prediction error value.

Feature descriptor supported gray image contains Histogram of Oriented Gradients (HoG), Scale Invariant Feature Transform (SIFT), speed up Robust Features (SURF) descriptors, which are used for compression, Pattern Recognition, Image Stitching etc. HoG [14] shows influences of various computation and summarized about methods orientation binning, coarse spatial binning, local contrast normalization for better result. SIFT [15] Scale invariant coordinates transform to its local features like key point localization, orientation assignments and key point descriptors. [16] shows different techniques under survey like Hashing, Transform coding and vector quantization and generates common framework called compressed HoG used for mobile image retrieval system. In SURF [17] scale and rotation invariant descriptor named SURF is presented, which compares for robustness, distinctiveness and repeatability. It works in two steps, it initially fixes the orientation to create a square region over selected orientation then extract descriptor format. In [18], it shows alternative technique for color to gray by using chrominance value called as single decomposition value.

As far as image retrieval is concerned, images are retrieved with different techniques. Starting from Text Based Image Retrieval (TBIR), which contains automatic or manual annotation of images. But representation of image in the form of text or wording is not sufficient and therefore study of Content Based Image Retrieval 
(CBIR) is introduced and it is described in the form of content of image like shape, color, texture etc. to avoid the description of images in the form of keywords, tagsand text. Multidimensional feature vector is used for representation of low level of feature of images. For representation of such visual content, a feature descriptor used, which again divided as local and global descriptors according to their extraction method.

For pre-processing of the images, Canny Edge Detection [19] used with different processing steps for removing noises and smoothing the images. As compared to other preprocessing techniques canny edge detection uses the double way of thresholding. It makes it more powerful in terms of finding true edges. Various kind of descriptors can be studied for images retrieval like SIFT, HoG, GF-HoG[20-22], Visual Content Descriptor etc, Edge Orientation Histogram(EOH) gives directions to gradients. ChanVeseModel[23] works to remove intensity inhomogeneity which occurs in medical images.

\section{PROPOSED SYSTEM}

The proposed work is combination of Image retrieval and Data retrieval. SBIR module contains edge extraction, descriptor generation and constraint application. This paper shows working of Data Hiding module. It contains Embedding process and Extraction process. Following Fig 1 shows flow of proposed work.

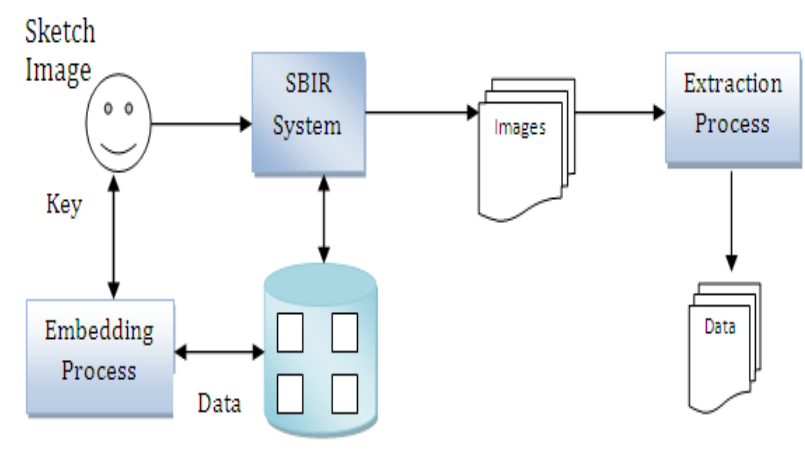

Figure.1 Proposed System Architecture

\subsection{Embedding Algorithm}

In color image data is embedded in Red, Green and Blue channel, where as in sketch image key is encrypted to extract the data at the receiver end. Grayscale version of image is important for further comparison of sketch and real image. Therefore grayscale invariance should be maintained throughout the embedding process till the extraction process.

\subsubsection{Division Algorithm}

This algorithm uses both sketch and color or real image. Which divides image into two parts, Insertable and Non insertablearea.Insertable area is further divided into smooth and complex regions by using algorithm [1]. It uses threshold value to find smooth and complex regions.

Step 1: Image $I$ is divided into insertable and non insertable areas. Non-insertable area contains extra information of properties, like threshold value, message length, key length and bit change information.

Step 2: Insertable area is again divided into smooth and complex regions depends on color intensity values. Data is embedded into smooth regions due to low intensity difference. Where $C_{i}$ is current reference pixel $\& C_{u}$, $C_{l}, C_{r}$ and $C_{d}$ shows remote central pixels on upper, left, right and down side respectively as shown in fig 2 .

$$
C_{i}=\left\{\begin{aligned}
\text { smooth }, & \text { difference }<\text { threshold } \\
\text { complex, } & \text { difference }>\text { threshold }
\end{aligned}\right.
$$


And differencevalue calculated by summation of differences between current reference pixel and remote central pixels.

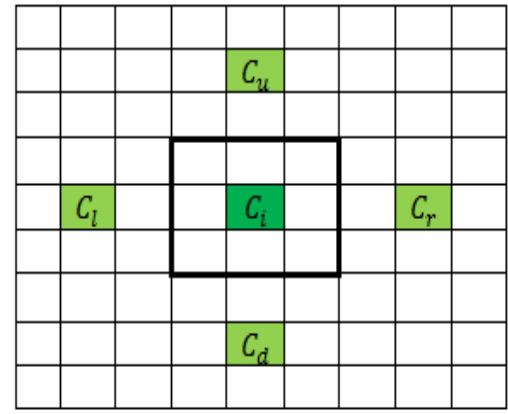

Figure.2 Reference pixel for data embedding

\subsubsection{For Sketch Image}

Input: Sketch Image (SI) of size A x B and key K, where A and B show height and width respectively.

Output: Sketch Image (SI') of size A x B with encrypted key.

Step 1: Extract Gray value from sketch image SI.

Step 2: Apply Division algorithm on image SI.

Step 3: Extracts the difference of pixel value by taking reference pixel for comparison. If the size of insertable area is $3 \times 3$,then central pixel denoted as $I_{C}$ as shown in fig. 3 which is compared with all even numbered pixels like $I_{2}, I_{4}, I_{6}$ and $I_{8}$.

\begin{tabular}{|l|l|l|}
\hline$I_{1}$ & $I_{2}$ & $I_{3}$ \\
\hline$I_{8}$ & $I_{C}$ & $I_{4}$ \\
\hline$I_{7}$ & $I_{6}$ & $I_{5}$ \\
\hline
\end{tabular}

Figure.3: Pixel Representation

Remaining pixels are compared with adjacent pixels with given direction as shown in fig 4. Resulting values are 0,1 and -1 as per difference value. The difference is calculated using equation (1) and (2) in which even values are 2, 4, 6 and 8 and odd values are 1,3,5 and 7according to pixels numbering and ' $\mathrm{d}$ ' shows the difference of adjacent pixels.

$$
\begin{gathered}
d_{\text {even }}=I_{\text {even }}-I_{c} \\
d_{\text {odd }}=I_{\text {odd }}-I_{\text {odd }+1}
\end{gathered}
$$

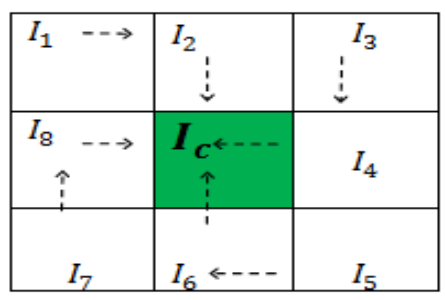

Figure.4: Pixel comparison 
Step 4: Difference histogram is created from values $d_{1}$ to $d_{8}$ of equation (1) and (2). Then extracted bit ' $\mathbf{c}$ ' from key $\mathrm{K}$ can be embedded by calculating difference of $d_{i} \& d_{i}{ }^{\prime}$.If secrete bit is extracted from the embedded data, can be embedded by modifying the difference value $d_{i}$ to $d_{i}{ }^{\prime}$.

$$
d_{i}^{\prime}=\left\{\begin{array}{cl}
d_{i} & \text { if } c=0 \\
d_{i} \pm 1 & \text { otherwise }
\end{array}---(3)\right.
$$

Scan the difference value $d_{i}$ sequentially, for $\mathrm{c}=1$, if difference value equals peak point of histogram then subtract 1 from $d_{i}$ else add 1 to $d_{i}$ as shown in equation 3 .

Step 5:Repetition of the step 4, until key is encrypted completely.

Step 6: Pixel values of encrypted sketch image (SI') calculated as shown in equation 4 and 5,

$$
\begin{array}{r}
I_{\text {odd }}=d_{\text {odd }}+I_{\text {even }}--------(4) \\
I_{\text {even }}=d_{\text {even }}+I_{c}
\end{array}
$$

Step 7: Finally, all blocks are combined to get encrypted image SI’.

\subsubsection{For Real Image}

Input: Real Image or Color Image (CI) of size A x B with Message M, where A and B are height and width respectively.

Output: Marked Color Image (CI') of size A x B.

Step 1: Extract Red, Green and Blue channels from color Image CI.

Step 2: Apply the division algorithm on color image.

Step 3: Compute difference for Red, Green and Blue channels respectively.

Step 4: Construct difference histogram.

Step 5: Repeat step 3 and step 4, for each channel until message is embedded completely.

Step 6: Calculate pixels of Color Image after message embedding.

Step 7: All blocks of all channels are combined to get marked color image (CI').

\subsection{Extraction Process}

It specifies the need of extraction method to be applied on sketch image and color image separately.

\subsubsection{For Sketch Image}

Input: Encrypted Sketch Image (SI') of size A x B

Output: Key k, Sketch Image SI".

Step 1: SI' is divided by applying division algorithm as explained above. LSB bit is extracted to retrieve key. It again divides the insert able area into smooth and complex regions.

Step 2: Compute the difference value $d_{i}$ ' by considering non-centered pixels.

Step 3: Scan the difference values in same order used previously. If the difference value is already recorded then skip it, then extract the key for next value of difference.

Original difference value $d_{i}$ is calculated as shown in equation 6 ,

$$
d_{i}=\left\{\begin{array}{cc}
d_{i}^{\prime} \pm 1 & \text { if } d_{i}=\text { in between } \\
d_{i}{ }^{\prime} & \text { Otherwise }
\end{array}\right.
$$

Value 1 is added in $d_{i}{ }^{\prime}$ if $d_{i}$ is in between peak values of histogram else subtract 1 from it.

Step 4: Repeat step 2 and 3 until key is completely extracted. 
Step 5: Construct Image SI'” as shown in equation 7 and 8.

$$
\begin{gathered}
I_{\text {odd }}=d_{\text {odd }}+I_{\text {even }}--------(7) \\
I_{\text {even }}=d_{\text {even }}+I_{c^{--------(8)}}
\end{gathered}
$$

\subsubsection{For Real Image}

Input: Embedded Color Image (CI') of size A x B

Output: Message M, Color Image CI'’.

Same algorithm of extraction mentioned above is applied for marked color image (CI') to retrieve the Message $\mathrm{M}$ and Color image (CI'). Message is extracted from all three channels. Step 2 and 3 from above algorithm is applied for each of three channels separately until data gets extracted completely.

\section{RESULTS}

The Data retrieval system contains data embedding and data extracting process, which is shown in below figures. Fig 5 shows grayscale conversion of selected image.

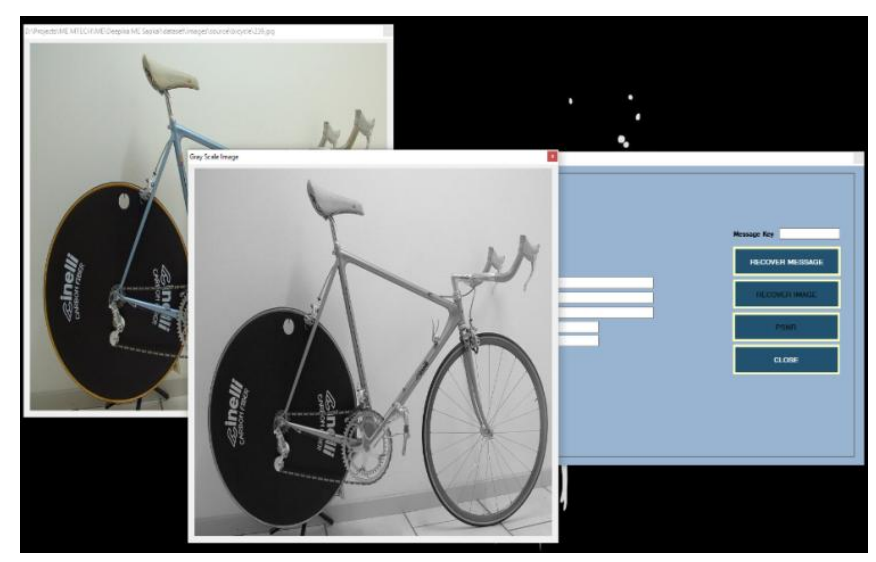

Figure. 5 Gray scale conversions

On this gray version Message embedding technique is applied, which shown by Fig. 6 .

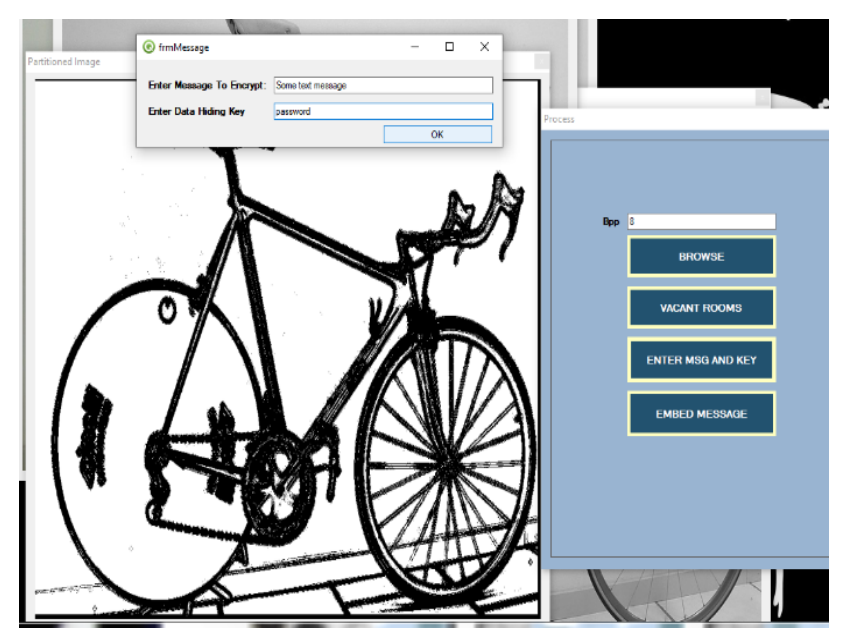

Figure.6 Message embedding in gray scale image. 
Next fig 7 shows image after message embedding in to image and fig 8 shows data extracting process from given image.

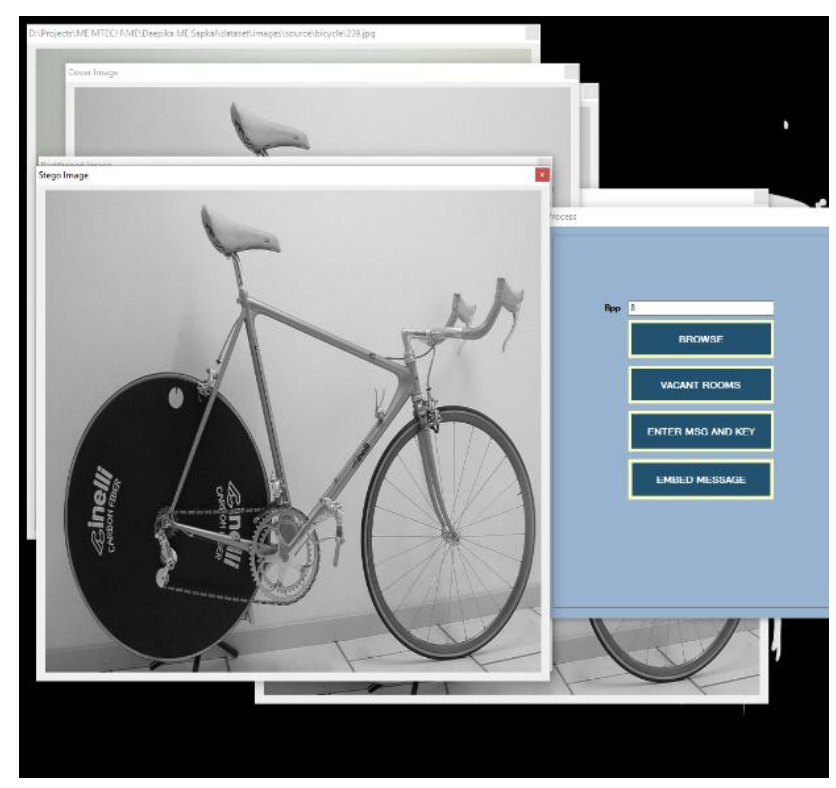

Figure.7 Image after Message embedding

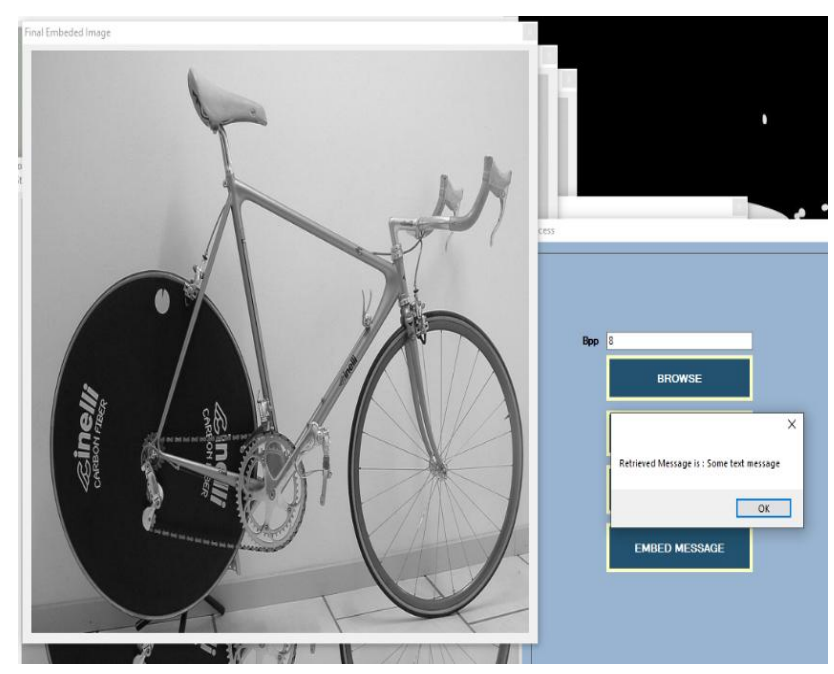

Figure.8 Message extracting from given image

\section{CONCLUSION}

This Data hiding algorithm is useful for both sketch and real image and ensures to maintain its gray-scale version throughout image processing. In embedding process, data is embedded in all three color channels due to which message length and embedding capacity is increased and gray-scale invariance is maintained. This Embedded sketch image and real image will be input for SBIR system, which will result in a novel proposed SBIDR system. 


\section{REFERENCES}

[1] R. DongdongHou, Weiming Zhang, Kejiang Chen, Sian-Jheng Lin and Nenghai Yu, "Reversible Data Hiding in Color Image with Grayscale Invariance.", in IEEE Transaction on circuits and Systems for Video Technology, 1051-8215, 2018.

[2] Shu Wang, Jian Zhang, Tony X. Han and Zhenjiang Miao, "Sketch Based Image Retrieval Through Hypothesis-Driven Object Boundary Selection With HLR Descriptor," IEEE transactions on Multimedia, Vol. 17, No. 7 July 2015.

[3] J. Fridrich and M. Goljan, "Lossless Data Embedding for All Image Formats," in SPIE Proceedings of Photonics West, Electronic Imaging, Security and Watermarking of Multimedia Contents, vol. 4675, pp. 572- 583, San Jose, Jan. 2002.

[4] J. Tian, "Reversible Data Embedding Using a Difference Expansion," IEEE Trans. Circuits System and Video Technology, vol. 13, no. 8, pp. 890-896, Aug. 2003.

[5] M. Kutter and S. Winkler, "A vision-based masking model for spread- spectrum image watermarking,” IEEE Trans. Image Processing, vol. 11, no. 1, pp. 16-25, Jan. 2002

[6] $P$. Bao and X. Ma, "Image adaptive watermarking using wavelet domain singular value decomposition," IEEE Trans. Circuits and Systems for Video Technology, vol. 15, no. 1, pp. 96-102, Jan. 2005.

[7] M. Asikuzzaman, M. J. Alam, A. J. Lambert, and M. R. Pickering, "Imperceptible and robust blind video watermarking using chrominance embedding: A set of approaches in the DT CWT domain," IEEE Trans. Information Forensics and Security, vol. 9, no. 9, pp. 1502-1517, Sep. 2014

[8] C. H. Chou and K. C. Liu, "A perceptually tuned watermarking scheme for color images," IEEE Trans. Image Processing, vol. 19, no. 11, pp. 2966-2982, Nov. 2010.

[9] Y. He, W. Liang, J. Liang, and M. Pei, "Tensor decomposition based color image watermarking," Proceedings of SPIE, vol. 9069, pp. 90690U- 90690U-6, Jan. 2014.

[10] M. R. A.Lari, S. Ghofrani, and D. McLernon, "Using curvelet transform for watermarking based on amplitude modulation," Signal, Image and Video Processing, vol. 8, no. 4, pp. 687-697, May 2014.

[11] C. Chang, C. Lin, Y. Fan, "Lossless data hiding for color images based on block truncation coding," Pattern Recognition, vol. 41, no. 7, pp. 2347-2357, 2008.

[12] J. Li, X. Li, and B. Yang, "Reversible data hiding scheme for color image based on prediction-error expansion and cross-channel correlation," Signal Processing, vol. 93, no. 9, pp. 2748-2758, 2013.

[13] B. Ou, X. Li, Y. Zhao, and R. Ni, "Efficient color image reversible data hiding based on channeldependent payload partition and adaptive embedding," Signal Processing, vol. 108, pp. 642-657, 2015. 
[14] N. Dalal and B. Triggs, "Histograms of oriented gradients for human detection," 2005 IEEE Computer Society Conference on Computer Vision and Pattern Recognition, San Diego, CA, USA, vol. 1, pp. 886-893, 2005.

[15] D. G. Lowe, "Distinctive image features from scale-invariant keypoints," Int. J. Comput. Vis., vol. 60, no. 2, pp. 91-110, 2004.

[16] Chandrasekhar V, Makar M, Takacs G, et al., "Survey of SIFT compres- sion schemes," In Proc. Int. Workshop Mobile Multimedia Processing, pp. 35-40, 2010.

[17] H. Bay, T. Tuytelaars, and L. J. V. Gool, SURF: Speeded up robust features, in Proc. Eur. Conf. Comput. Vis., pp. 404-417, 2008.

[18] Sowmya V, Govind D, Soman K P, "Significance of incorporating chrominance information for effective color-to-grayscale image conversion, "Signal, Image and Video Processing, vol. 11, pp. 129136, 2017.

[19] J. Canny, “A computational approach to edge detection,”' IEEE Trans. Pattern Anal. Mach. Intell., vol. PAMI-8, no. 6, pp. 679-698, Nov. 1986.

[20] Y H Sharath Kumara, D S Gurub, "Retrieval of Flower Based on Sketches," in International Conference on Information and Communication Technologies, Procedia Computer Science 46 (2015) $1577-1584$.

[21] R. Hu, M. Barnard, and J. Collomosse, "Gradient field descriptor for sketch based retrieval and localization, ' in Proc. IEEE Int. Conf. Image Process. (ICIP), Sep. 2010, pp. 1025-1028.

[22] R. Hu and J. Collomosse, "A performance evaluation of gradient field HoG descriptor for sketch based image retrieval.” in Comput. Vis. Image Understand., vol. 117, no.7, pp. 790806, 2013.

[23] Tingting Liu, HaiyongXu, "Medical Image Segmentation Based on a Hybrid Region-Based Active Contour Model”.

[24] SN Popat, YP Singh, "Efficient Research on the Relationship Standard Mining Calculations In Data Mining” in Journal of advances in Science and Technology, Volume14, Issue No.2, September 2017.

[25] SN Popat, YP Singh,"Analysis and Study on The Classifier Based Data Mining Method" in Journal of advances in Science and Technology, Volume14,Issue No.2,September 2017.

[26] Prof. S.T. Khandare et al, "A Survey Paper on Image Segmentation with Thresholding”, International Journal of Computer Science and Mobile Computing, Vol.3 Issue.1, January-2014, pg. 441-446. 